\title{
A Focus Group Research on the Use of Wearable Health Technologies in Rehabilitation
}

\author{
Pınar Pektekin ${ }^{1}$, Hasan Kerem Alptekin ${ }^{2}$, Halil İbrahim Ural ${ }^{3}$, Mirsad Alkan ${ }^{4}$, \\ Hatice Borac1 ${ }^{5}$ \\ ${ }^{I}$ Assistant Professor, Bahcesehir University, Management of Health Institutes Department, \\ Health Sciences Faculty \\ ${ }^{2}$ Assistant Professor, Bahcesehir University, Physiotherapy and Rehabilitation Department, \\ Health Sciences Faculty \\ ${ }^{3}$ Assistant Professor, Bahcesehir University ,Physiotherapy and Rehabilitation Department, \\ Vocational School of Health Services \\ ${ }^{4}$ Research Assistant, Bahcesehir University, Physiotherapy and Rehabilitation Department, \\ Health Sciences Faculty \\ ${ }^{5}$ Lecturer, Bahcesehir University, Physiotherapy and Rehabilitation Department, \\ Health Sciences Faculty
}

\begin{abstract}
Wearable technologies are becoming more and more effective thanks to what they promise. The conversion of these technological developments into the devices which can be used in health industry has ensured a common ground for informatics and health specialists. The study depicted in this article was conducted for the use of wearable health technologies in rehabilitation. The perspectives, attitudes, behaviours and expectations of the participant individuals were researched and it was intended to obtain such findings that could direct new technologies. The focus group method established by six specialist academicians was employed for this study. The findings were reported as written records. A discussion chapter was also included in the article to provide an insight for future studies.
\end{abstract}

Keywords: Health informatics, wearable technology, rehabilitation, virtual reality, the use of technology

\section{Introduction}

Recent technological developments evolve and transform both the business and private life. Thanks to the developing technology, organ designs can be performed; pulses can be checked and any possible health problems can be predetermined through smart watches; old people can be warned against falling down with the help of the devices they wear; fingers and organs (soon) can be produced and these reveal the infiniteness of the dimensions of the transformation in health industry. Some examples are available like orientation through the shoes being the products of the wearable technology for the handicapped individuals or the ones in a rehabilitation process or prevention of collisions with approaching objects with the help of the clothes equipped with sensors. The fears of handicapped individuals to go out or arrive in any location have been minimized thanks to these technologies. The need of these individuals for help by others to go on their lives can be minimized through wearable technologies. This is a quite important development for mental and physical health of the handicapped. The development of robotic systems facilitates the life of orthopaedic patients and the developments in robot technologies will feel the emotions of individuals and provide assistance for the psychology and health of these individuals. For instance the capability to detect whether an individual is crying for pain or because of an emotional reason will be more effective in communication between machines. The effect of painkillers in smart pillboxes or on the prescription given by a doctor, the time of taking the same, detection of their severity and the identification of attitudes and behaviours based on the approval of the server robot show fast acceleration of these technologies.

A focus group research was conducted on the use of wearable health technologies in rehabilitation within the scope of this study. Current situation was revealed on the basis of the findings obtained at the end of the study and a discussion chapter was included at the end of the study to provide an insight for future studies with the data available.

\section{Wearable Technologies}

The robotic systems used in rehabilitation are mainly mobilization systems, virtual reality systems, upper extremity robots, walking lokamats, robotic orthesis, myoelectric prosthesis, exoskeletons etc. Before dealing with the operation of these systems, we must explain that the patient population for whom these systems are preferred is generally neurological patients. Robotic systems can also be used for orthopaedic problems. 


\section{Mobilization Systems}

The mobilization systems used in hospitals are generally the systems deployed in intensive care units and electrical tilt tables and the robotic systems acting as a lifter draw attention as the primary preference. However, neither system can be used in several intensive unit cares due to their large dimensions and the difficulty in mobile usage under intensive care unit conditions.

\section{Virtual Reality Systems}

The term of virtual reality will remind of game consoles that have become quite popular today for many of us. The sports and action games which have recently become widespread and are fondly preferred by people perceive the movements of people with the help of sensors and ensure entertainment by making people feel as if they were the actual players. Additionally, it is obvious that patients can experience the same joy and get better if the same game software is used in rehabilitation. Moreover, it must be specified that virtual reality software increases the adaptation of patients in many rehabilitation robot and facilitate the operation of it.

\section{Brain-Machine Interface (BMI)}

Brain machine interface systems developed as a result of the studies conducted in this area which protect their popularity in recent years and intended for allowing people to control machines through their brains are also highly important for wearable technologies. The objectivity of scientific studies has increased as the availability of these systems enhances and these systems become widespread and the development of robotic rehabilitation has accelerated significantly. Furthermore, these systems are being used in treatments as a part of robotic rehabilitation. The system operates under the principle that the data obtained from the brain through Electroencephalogram (EEG) are analyzed and the inputs obtained from such analysis are subjected to a second processor and converted into a command for the machine. When the mode of operation is basically reviewed, it is understood that it transmits electrical signals received from the neurons to machines in such a way that it will reveal motor movement. Although the mode of operation seems very simple, there are still many steps required to be taken particularly for the solution of operation related problems. Scientific studies suggest that the devices used in brain machine interface must be calibrated in a customized manner. It was concluded in a study conducted on the individuals with and without a brain damage that the individuals with a brain damage had undergone more difficulty in the operation of the machine than the individuals without any brain damage. Additionally, the complexity of the emotions and feelings in human brain having a complicated nature can also make the operation difficult.

\section{Upper Extremity Robots}

The system is similar to the brain machine interface explained in the description of the virtual reality concept. A communication between the robot and human is ensured with combined use of Functional Electrical Stimulation (FES) and Electromyogram (EMG) methods employed in diagnosis, neurological rehabilitation and some orthopaedic rehabilitation applications and patients are subjected to rehabilitation under the neurofeedback method. Upper extremity robots resulting from the combination of robotic orthesis with neurofeedback and virtual reality applications serve for the humankind in many areas.

\section{Walking Lokomats}

Lokomat systems consisting of a computer processor which ensure the communication of robotic long walking orthesis, patient weight bearing system, treadmill and machine parts with each other and with the patient and physiotherapist and provide support for the virtual reality software rehabilitate patients under the relearning model and protect patients against the complications of long term confinement to bed by means of performing the walking activity in a passive manner for patients who cannot be rehabilitated.

\section{Robotic Orthesis}

The systems consisting of accumulators acting as a power source for orthesis devices, robotic arms, superficial electromyography equipment receiving stimulants from patients, sensors receiving environmental stimulants and a processor calibrating the orthesis in a customized manner are called as robotic orthesis. In addition to the movement strategies in early orthesis, it perceives the signals sent to muscles by neurons with the electromyography (EMG) device and transmits these signals to the processor and robotic arms are used to direct the orthesis to contribute to movement after the data are processed by the processor $(2,3)$. In this way, patients become capable of doing the movements which they cannot or have difficulty in doing. The patient is warned against extremely cold or hot conditions which cannot be felt by patients due to the loss of sense and may damage the extremity through the stimulants received from the environment with the help of the sensors attached to some orthesis or otherwise the movement is stopped by the robotic systems. For instance patients cannot hold a pot which involves the risk of burning hand through the use of the robotic system. 


\section{Exoskeleton Robots}

Wearable robots resulting from the combination of multiple robotic or thesis on a single robotic plane are called as exoskeleton. These systems act as an external skeleton and support the body and help people move. Data inputs are received in this system not only from the stimulants for the muscles but also from spinal cord levels, spinal cord or the part of brain related to the central nervous system differently from robotic or thesis. However, the problems of the system particularly related to balance have not been solved yet and for this reason, patients need a supplementary assistant device (i.e. forearm crutch) even though they wear exoskeleton.

\section{Myoelectric Prosthesis}

Another system which has been maintaining its currency for a long period of time and whose development still carries on is myoelectric prosthesis. These systems perceive what patients want to do with the help of EMG electrodes placed on the muscles of patients and then perform that movement by imitating a real hand through its own power source and robotic mechanism. Even if quite successful models in terms of image have been developed and such myoelectic prosthesis that can perform about 20-30 different hand movements have been designed, they are still not found sufficiently qualified in terms of usage and fail to satisfy patients enough. Still, they are undoubtedly necessary owing to their advantages which ensure that patient get tired less while they are performing the tasks either in terms of function or less effort when they are compared with standard prosthesis mechanisms.

\section{Use Of Wearable Health Technologies In Rehabilitation}

Today, we co-exist with robots that fulfil important duties for our lives. Even though the most frequently used type of these robots is generally industrial robotic arms, we encounter with the designs really enhancing the quality of life in health industry. The developments in industrial and technological areas take effect also in the health industry. Diseases can be diagnosed earlier, surgical operations can be overcome with less risk and rehabilitation can be completed in a shorter period of time in case of disability thanks to these developments.

\section{Mobilization Systems}

Patients' being able to get out of bed at an early phase following the disease even at intervals makes significant contribution for the regulation of their respiration functions and blood pressure; reduces the occurrence of atrophy on their muscles due to passivity and assists in shortening the rehabilitation process and getting back to daily life for patients in a shorter period of time by way of protecting patients against multiple comorbidity appearing in the rehabilitation process.

An exercise program was applied for the patients followed in the intensive care unit with a Cerebral Vascular Accident (CVA) story in such a way that they could take 38-40 steps per minute for 20-30 minutes a day with the combination of a tilt table and stepper within the scope of a study conducted by Russian clinicians in 2012. At the end of the study, the blood flow, blood pressure and muscular leg force of the patients made better progress than the patients for whom no tilt table and stepper had been used (1).

We can summarize the advantages offered to patients by early mobilization against the possibility of long term immobilization in a number of articles:

1) Functional skills of the patients rehabilitated with a robotic system following a Cerebral Vascular Accident (CVA) make better progress than other patients because the blood flow in their brand returns to normal earlier.

2) One of the critical problems faced during the mobilization process after a long period of immobilization is orthostatic hypotension. Early stage mobilization is highly important in the prevention of orthostatic hypotension in patients. Robotic systems play a vital role at this point and provide great convenience both for patients and therapists.

3) Patients feel less weakness at the time of mobilization and walking as a result of the programs ensuring functional movements by activating many muscle groups simultaneously with robotic systems for the recovery of muscle atrophies related to long term immobilization and thus the rehabilitation period shortens and patients can get back to their normal lives earlier.

4) Such complications as bone density loss, pressure sores, occurrence of deep venous thrombosis, infections resulting from the problems in lung hygiene etc. can be prevented with proper mobilization of patients and the scenarios leading to loss of life can be avoided. Additionally, walking exercises performed with walking robots ensure the operation of the systems in the body and prevent the abovementioned complications even if the patient will not recover. 


\section{Virtual Reality Systems}

Virtual reality software plays an important role in rehabilitation because of the fact that they promote hand and eye coordination; improve hand skills; develop the ability to balance and increase functional independence with the combination of all these advantages. Virtual reality systems are used in the prevention of existing skills or the improvement of skills in the rehabilitation of multiple sclerosis (MS), Parkinson's disease, cerebral palsy etc. in addition to CVA (7).

\section{Brain-Machine Interface (BMI)}

Brain machine interfaces are not regarded as treating or rehabilitating systems; however, they are quite important in the adaptation of patients to rehabilitation and particularly to robotic rehabilitation systems and their basic function is to help rehabilitation. Many scientific studies have proved that they helped rehabilitation in cases of speech disorders called as aphasia which result from damages on some brain areas responsible for tongue; autistic disorders involving motor function disorders; neurodegenerative diseases including motor disorders like Parkinson's disease and spinal cord injuries. Although they are estimated to be successful in use for the patients suffering from amyotrophic lateral sclerosis (ALS), it is stated that no study has been conducted on this regard yet due to less number of patient population in comparison with other disease groups or that these studies have not been observed in literature.

\section{Upper Extremity Robots}

Upper extremity robots are commonly preferred in the rehabilitation of hemiplegic arm resulting from CVA, function losses on upper extremity as a result of spinal cord injuries, problems of neurologic origin such as coordination disorders resulting from Parkinson's disease and cerebral palsy as well as such orthopaedic problems as joint and muscle sclerosis, muscle weakness, muscle spasm etc. and the movement disorders caused by these problems. Reflex Sympathetic Dystrophy Syndrome (RSD) appearing on the distal of the extremity where the disease is diagnosed is a state which not only delays the recovery period of the movement expected to recover in the rehabilitation process but also prevents these movements for some individuals suffering from these diseases. Less Reflex Sympathetic Dystrophy Syndrome (RSD) was encountered in the patients subjected to robotic rehabilitation in comparison with the patients not subjected to it as a result of the studies conducted; robotic rehabilitation also has a protective effect on patients from this aspect.

Robotic rehabilitation used particularly in combination with neuro-feedback and virtual reality has been stated to make greater contributions to the rehabilitation process by making a superior effect than other methods in the studies comparing traditional methods employed for upper extremity rehabilitation with robotic rehabilitation among recent meta-analysis studies.

\section{Walking Robots}

Many robotic rehabilitation systems particularly walking robots re-teach the movements which cannot be performed by patients with the use of a limbic system for patient rehabilitation. For this reason, it is highly probable that these systems to fail in case of damages on the limbic system especially the hippocampus during any brain injury. Studies have suggested that the ability of walking robots to increase limbic system warning improves blood flow in the brain and the cognitive level (11). Moreover, it has been stated that the use of walking robots ensure a real like walking and sole pressure distributions are close to the real values when the walking analyses and sole pressure distributions with and without walking robots are compared and that particularly the sense of proprioception and other senses recover earlier in this way (5). Furthermore, it must be noted that the walking exercise applied will strengthen muscles and prevent muscle atrophies. A systematic compilation has established the hypothesis that walking robots have positive effects on balance and they improve balance as a result of all these factors and the hypothesis has been confirmed with the articles compiled within the scope of the study (4). It has also been suggested many times that positive effects are observed on the heart, lung and circulation system of patients doing exercises with walking robots and their movement capacities are enhanced.

\section{Robotic Orthesis, Exoskeleton, Myoelectric Prosthesis}

Thanks to these systems, many individuals confined to wheeled chairs or bed can walk at least with the help of crutches and get back to their daily lives. Robotic orthesis is generally used on certain extremity or joint of the body such as the knee joint which cannot fulfil its functions due to lower leg, plegia or paretic extremity, muscle weakness or various reasons whereas exoskeleton systems are regarded as a promising product for the patients who are confined to bed particularly because of complete spinal cord injuries and they can provide a protective health service thanks to their use in the performance of heavy works by industrial workers.

Myoelectric prosthesis, on the other hand, is regarded as robotic systems which can be used for the individuals who surgically lost their extremity (amputation) due to any accident or various reasons and perform 
some fine motor activities and rough movements of hand especially in case of lower elbow amputations even though they have not been developed enough to represent full arm or leg. It can be accepted as a part of rehabilitation because of the fact that any restricted function due to the loss of extremity can be offered to the amputated patient even with the help of mechanical systems. On the other side, myoelectric prosthesis has been produced to be used also for lower knee amputations as a result of recent R\&D studies.

\subsection{Scope of the Research}

\section{Research}

\section{a. Subject of the Research}

The subject of the research is to identify the technological areas and applications which can affect the developments within the scope of the use of wearable health technologies in rehabilitation and determine the priorities and expectations of health and informatics specialists with the effect of evolving treatment environment.

\section{b. Research Questions}

The questions which have been designated in consideration of the subject and purpose of the research and tried to be answered are presented below.

Research Question 1 (RQ-1): Is the ease of use of technologies important for you?

Research Question 2 (RQ-2): What are your opinions about the use of wearable health technologies?

Research Question 3 (RQ-3): What is your perspective for rehabilitation with the help of wearable technologies in case of disability?

Research Question 4 (RQ-4): Do you think that the use of robotic systems which are the products of developing health technologies in today's world in orthopaedic problems made an effective difference?

Research Question 5 (RQ-5): What are your opinions about the use of virtual reality systems in the rehabilitation of patients suffering from disorders in their central nervous system?

Research Question 6 (RQ-6): What are the areas and applications of technology that will trigger and accelerate any possible technological developments in wearable health technology devices?

\section{c. Importance of the Research}

Focus group studies provide comprehensive and multidimensional qualitative data about the tendencies, opinions, emotions, attitudes and habits of the target audience on a predetermined issue. This article involves the activities related to the establishment of an awareness level with respect to the beliefs, attitudes and behaviours of specialists in the use of wearable health technologies in rehabilitation and direction of the same if required and aims at revealing how the participants perceive the subject.

\section{d. Population}

The most important advantage of focus group discussions is the appearance of new and different ideas as a result of in-group interaction and group dynamics. Participants trigger the emotions and thought in the minds of each other as a result of mutual association and this ensures various and rich information flow. It also provides the opportunity of getting access to rich and various information in case of a time and budget limitation. The population of this study is a group consisting of computer engineers, medical doctors and physiotherapists.

\subsection{Research Methodology}

Descriptive and qualitative research methods were employed in the study respectively for the purpose and method. The methods of focus group study were used as a means of data collection with the non-structured in-depth interview technique which is frequently preferred in qualitative research method.

Conceptual and theoretical framework was established in relation with the term of wearable health technologies and the plan was made accordingly.

\section{At this stage:}

- The specialists were selected for the interviews in consideration of the equal distribution in the areas of computer and health sciences.

- The underlying reason of the selection of these individuals is that they have received education and conducted studies in this field.

- Common characteristic of the selected individuals is that they have done researches on the subject matter.

- The titles identified for the interview were selected in accordance with the use of wearable health technologies in rehabilitation.

- Order of the questions is from the general to the specific in terms of the concepts used in the research. 
The time, location and the observer who would take down notes during the interview were designated and the focus group interview was started at the designated time and location. Following the interview, short notes taken down by the observer were collected in consideration of the characteristics of the social surroundings including the group. The equilibrant was ensured by way of allowing none of the participants to dominate the group or remain recessively. Some interesting sentences of the participants were subsequently taken and the study was reported.

\section{Conclusion}

The data obtained from the research was reported in this chapter. The responses provided by the individuals participating in the focus group study were regarded and interpreted as findings in the preparation of such report. Findings cover the analysis of research questions respectively following the provision of demographical information.

\subsection{Results}

We can summarize the findings obtained with the focus group method within the scope of the study as follows:

The focus group consists of 3 female and 3 male participants in the age range of 25-40. Three participants are academicians with a doctorate degree and a specialty in the field of informatics and they are capable of performing critical analysis of the subject matter.

When the responses of the participants to the question of "Is the ease of use of technologies important for you?" were analyzed, the participants stated that the availability of functional properties by people had been as important as functionality even though the simplest way to facilitate our lives with technology was to produce functional and accessible technologies. Otherwise, technologies would not serve a purpose for the humankind despite of their development. On this regard, they defended the perspective that ease of use of the produced technologies was highly important. They also reported that ease of use of technologies had been observed to be important in the field of rehabilitation and user-friendly and simple technologies has been preferred to complex ones at the advanced age group (65 and above) with slight difference in the population who was closer to these technologies and used the same more frequently. They even expressed that the concept of exergame combining the games in game consoles with exercise had recently been becoming popular and these types of console games and biofeedback devices had been useful particularly in balance rehabilitation of old patients within the scope of several systematic compilations.

When the responses of the participants to the question of "What are your opinions about the use of wearable health technologies?" were analyzed, the participants stated that technology had reminded of power and a more comfortable life in today's world; the concept of power in the field of health had provided the meaning for individuals of muscular power and the power to carry on the duties required to be performed to sustain their lives and for this reason, the material to be used had to comply with the system first to recover their lost skills due to various diseases with technological support. Finally, it is required to highlight that the accessibility of technologies is as important as their ease of use. The participants suggested that wearable technologies had been a recently emerging and promising area in health industry; the investments in this area would increase day by day and especially the difficulty or cost increases in people's access to health systems had supported the studies on this regard.

Furthermore, the participants specified that preventive medicine had come to the foreground in this area and there had been some studies related to posture correction and exercise arrangement with wearable technologies which involved the wearable technologies called as biosensor for the prevention of pressure sores due to long term immobilization or wearable smart T-shirts for instant detection of any heath disease with electrocardiogram monitoring.

When the responses of the participants to the question of "What is your perspective for rehabilitation with the help of wearable technologies in case of disability?" were analyzed, the participants stated that wearable technologies used in case of disability could remove negative effects of this situation and play the role of integrating individuals into the social life for some times and they could be used as a part of the treatment process for some other times. They also reported that in both cases, mentioned technologies had facilitated the life if individuals and formed a basis for living together with healthy individuals again by holding on to life and individuals could not only be saved from confinement to bed or wheeled chair with the help of these technologies but also they could perform self-care and daily life activities either independently or semidependently. One of the participants additionally explained that the wearable technologies could act as such systems that monitor and warn the patient against various hazards in case of disability or discover early symptoms of some diseases and the highest number of studies had been conducted on old people and the situation of falling down on this regard and the systems calculating downfall related risks faced by old people and warning them by using various sensors had recently been in use. Another participant noted that wearable technologies with the purpose of direct treatment had been available; individuals could perform some 
movements that they were unable to do with the support of glove shaped exoskeletons and the same system had been applied to whole body exoskeletons.

When the responses of the participants to the question of "Do you think that the use of robotic systems which are the products of developing health technologies in today's world in orthopaedic problems made an effective difference?" were analyzed, the participants reported that the devices designed for use by patients in their daily lives had been insufficient in terms of ease of use and success despite the fact that serious developments had been achieved in the field of orthopaedic rehabilitation in general. They stated that the underlying reason had been that the movements performed by patients in their daily lives particularly in case of hand problems could not be fully integrated into robotic systems and there had been some problems in feedback processes and for this reason, satisfactory achievement for patients had not been ensured yet despite of the fact that serious progress was made. They also explained that the success of the robots used in orthopaedic rehabilitation had been very satisfactory and their positive effects had been proven in several scientific studies. One of the participants noted that these types of robotic systems had been started to be used with the deployment of weight transferring treadmills in the treatment of patients with spinal cord injury or hemiplegia and it was detected that the rehabilitation applied with these treadmills had adapted patients to daily life activities in a quicker and safer manner than conventional rehabilitation and walking training and that the robotic haptical devices developed upon such detection had determined the pressures applied by individuals and provided muscle support to them at that level. Moreover, they expressed that these devices had generally been connected to the treadmill but created a biofeedback mechanism on robotic walking sections and on the area where patients walked through virtual reality and that exoskeletons had been attached to end of these devices and there had been some instances showing that the individuals with spinal cord injury could do up and down stairs at certain levels thanks to these wearable technologies.

When the responses of the participants to the question of "What are your opinions about the use of virtual reality systems in the rehabilitation of patients suffering from disorders in their central nervous system?" were analyzed, the participants explained that they had considered that virtual reality systems which increased especially limbic system activity in case of the disorders observed in central nervous system and made significant contributions to restart of motor learning created positive effects in many diseases; enhanced reality applications allowed by our current technological capability had created the same effect with virtual reality applications even though different positive effects of virtual reality applications were revealed in various scientific studies and on the top of it, they had been proved to be more successful in creating these effects than virtual reality according to the studies conducted. The participants also expressed that the patients for whom virtual reality was used for the first time in this area had been the patients who lost their upper or lower extremity functions following hemiplegia or suffered from cerebral palsy; it had been subsequently started to be used in spinal cord injuries, amputated rehabilitation and even vertigo rehabilitation and that minimum 10 sessions had to be applied for hemiplegia patients when the studies on virtual reality and enhanced reality were reviewed. They defended that the advantage of this study had been that patients did not get bored in long exercise durations and worked at a high motivation level with the use of dramatization theory after a close environment to their daily life activities was created. One of the participants, on the other hand, reported that Einstein's formula for success had been Formula $A=X+Y+Z$ ( $A=$ Achievement, $X=E f f o r t, Y=$ Regarding/enjoying the issue as a game, $\mathrm{Z}=$ Producing instead of speaking) and that this had explained the success of virtual reality in rehabilitation.

When the responses of the participants to the question of "What are the areas and applications of technology that will trigger and accelerate any possible technological developments in wearable health technology devices?" were analyzed, the participants reported that the most important problem in wearable health technologies had generally been uncomfortable and heavy products and short battery lives and the EMG sensors except these had sometimes been insufficient and time losses encountered during the feedback process had made usage more difficult; some developed wearable devices could compensate these defects through their functionalities and they had considered that the development of wearable technological products would reach to higher levels together with the progress in the disciplines helping to understand the functioning of human body such as neurology and biomechanics especially in addition to metallurgy and materials, electrics and electronics, machines, robotic and software. Moreover, the participants suggested that the increase in the relationship cycle between individuals and health personnel through wearable health technologies would allow new treatment methods in this area; the findings related to the slightest nerve compression could be notified through an early warning system upon the adaptation of wearable technologies to the whole body, muscles and nerves and that the muscles tending to injure during technology use or doing sports could be featured and intervened with before they appear. 


\section{Discussion}

The contribution of technological developments to the humankind plays a vital role when the issue is handled with a wide perspective. Technology has become indispensable for human beings thanks to the facilities it offers for daily life; its importance in the protection of human life and its ability to ensure the recovery of lost skills. The availability and accessibility of technology is more important than the development level of the produced technology as stated both in literature and expert opinions. Easier accessibility and availability of technology varies depending on the development level of it.

When the technological developments from past to present are reviewed, it can be seen that scientists who were in search of such systems that could be controlled with human brain set the cornerstones for robotic rehabilitation systems and wearable technologies.

As a basic problem, excessive area occupancy of systems based on their functions; insufficiency of produced technologies of reasonable dimensions due to battery life, balance etc.; inability of the human machine interfaces to provide desired efficiency in a complete manner due to mental state or damaged tissues in brain and consequently the unavailability of technologies are the obstacles for their full integration. Still, less area occupancy of the devices, rapid operation of complex systems and most importantly the ability to figure out complicated issues related to human beings one by one integrates robotic systems in our lives more and more thanks to the developments in materials science, engineering science and health sciences.

The most important advantage of the combination of the techniques employed in conventional rehabilitation applications with robotic systems in groups is lower costs with the shortening of the period of treatment and return to daily life. Shortening the hospitalization period and accelerating the period of return to work prevent serious monetary and non-monetary losses. At this point, we can say that rehabilitation technologies answer for the costs paid to them to the fullest extent.

Consequently, it is obvious that positive aspects of wearable technologies used particularly in health area outweigh when they are evaluated with all positive and negative aspects. Even though R\&D studies required for the removal of negative aspects necessitate high budgets, these systems will be able to afford R\&D costs in the long run thanks to their benefits.

\section{References}

[1]. Alexey N. Kuznetsov, Natalia V. Rybalko, Vadim D. Daminov, and Andreas R. Luft; Early Post-stroke Rehabilitation Using a Robotic Tilt-Table Stepper and Functional Electrical Stimulation ; Stroke Research and Treatment; Volume 2013; p. 1-9

[2]. Nathanaël Jarrassé, Tommaso Proietti, Vincent Crocher, Johanna Robertson, Anis Sahbani, Guillaume Morel and Agnès RobyBrami; Robotic exoskeletons: a perspective for the rehabilitation of arm coordination in stroke patients; December 2014; Volume 8; p. $1-13$

[3]. Ho Shing Lo, Sheng Quan Xie, Exoskeleton robots for upper-limb rehabilitation: State of the art and future prospects; Medical Engineering \& Physics 34 (2012); p. 261-268

[4]. Eva Swinnen, David Beckwée, Romain Meeusen, Jean-Pierre Baeyens, and Eric Kerckhofs ; Does Robot-Assisted Gait Rehabilitation Improve Balance in Stroke Patients? A Systematic Review; Top Stroke Rehabil 2014;21(2); p.87-100

[5]. Stefan Hesse, Henning Schmidt, Cordula Werner, Anita Bardeleben; Upper and lower extremity robotic devices for rehabilitation and for studying motor control; Current Opinion in Neurology 2003, 16; p. 705-710

[6]. Won Hyuk Chang, Yun-Hee Kima; Robot-assisted Therapy in Stroke Rehabilitation; Journal of Stroke 2013;15(3); p. 174-181

[7]. Yao-Jen Chang, Ya-Shu Kang, Po-Chiao Huang; An augmented reality (AR)-based vocational task prompting system for people with cognitive impairments; Research in Developmental Disabilities 34 (2013); p.3049-3056

[8]. Maarten RC van den Heuvel, Erwin EH van Wegen, Cees JT de Goede, Ingrid AL Burgers-Bots, Peter J. Beek, Andreas Daffertshofer, Gert Kwakkel; The effects of augmented visual feedback during balance training in Parkinson's disease: study design of a randomized clinical trial; BMC Neurology 2013; 13:137

[9]. Fernando E. R. Mattioli, Edgard A. Lamounier Jr., Alexandre Cardoso, Alcimar B. Soares, Adriano O. Andrade; Classification of EMG signals using artificial neural networks for virtual hand prosthesis control; 33rd Annual International Conference of the IEEE EMBS Boston, Massachusetts USA, August 30 - September 3, 2011

[10]. Pawe B. Kiper, Michela Agostini, Carlos Luque-Moreno, Paolo Tonin, Andrea Turolla; Reinforced Feedback in Virtual Environment for Rehabilitation of Upper Extremity Dysfunction after Stroke: Preliminary Data from a Randomized Controlled Trial; BioMed Research International; Volume 2014

[11]. Michael Villiger, Patrick Grabher, Marie-Claude Hepp-Reymond, Daniel Kiper, Armin Curt, Marc Bolliger, Sabina HotzBoendermaker, Spyros Kollias, Kynan Eng, Patrick Freund; Relationship between structural brainstem and brain plasticity and lower-limb training in spinal cord injury: a longitudinal pilot study; Frontiers in Human Neuroscience; 1 May 2015; Volume 9; 254

[12]. Michael Villiger, Dominik Bohli, Daniel Kiper, Pawel Pyk, Jeremy Spillmann, Bruno Meilick, Armin Curt, Marie-Claude HeppReymond, Sabina Hotz-Boendermaker, Kynan Eng ; Virtual Reality-Augmented Neuro-rehabilitation Improves Motor Function and Reduces Neuropathic Pain in Patients With Incomplete Spinal Cord Injury; Neuro-rehabilitation and Neural Repair 27(8); 675683 